\title{
Smoothed particle hydrodynamics modeling of viscous liquid drop without tensile instability
}

\author{
Xiufeng Yang*, Moubin Liu, Shiliu Peng \\ Institute of Mechanics, Chinese Academy of Sciences, Beijing 100190, China
}

\section{A R T I C L E I N F O}

\section{Article history:}

Received 24 July 2013

Accepted 7 January 2014

Available online 11 January 2014

\section{Keywords:}

Liquid drop

Smoothed particle hydrodynamics

Tensile instability

\begin{abstract}
A B S T R A C T
Smoothed particle hydrodynamics (SPH), as a Lagrangian meshfree particle method, has been applied to modeling viscous liquid drop with surface tension and wetting dynamics. In the SPH model, the van der Waals (vdW) equation of state is usually used to describe the gas-to-liquid phase transition similar to that of a real fluid. However, the attractive forces between SPH particles originated from the cohesive pressure of the vdW equation of state can lead to tensile instability, which is associated with unphysical phenomena such as particle clustering or blowing away. This paper presents an improved SPH method for modeling viscous liquid drop. The inherent tensile instability in SPH is removed by using a hyperbolicshaped kernel function which possesses non-negative second derivatives. A single-step approximation for heat flux is used in modeling viscous liquid drop with smoother temperature field. The formations of viscous liquid drops, both in 2D and 3D, are tested and it clearly demonstrates that the tensile instability can be effectively removed. The improved SPH method is also used to model two other numerical examples including the oscillation and binary collision of liquid drops without tensile instability.
\end{abstract}

(ㄷ) 2014 Elsevier Ltd. All rights reserved.

\section{Introduction}

Liquid drop dynamics has been investigated for many years as liquid drops exist widely in nature and industrial production, such as the formation of raindrop, ink-jet printing, fuel injection atomization, droplet-based net-form manufacturing, and many others [1]. Liquid drops were firstly studied quantitatively by Rayleigh [2], and he derived the relation between the period of drop vibration and the surface tension. In the past few decades, Liquid drops have been experimentally investigated and numerical modeled by many researchers. For example, in order to study the rheological properties of liquid drop surfaces, Apfel et al. [3] studied the free oscillations and large deformations of water drops in microgravity. Willis and Orme [1,4] experimentally investigated the viscous binary droplet collisions in a vacuum environment. They quantitatively studied the oscillation cycle of droplets and found that the time period of oblate oscillation is relatively not affected by Weber number and viscosity and that the time period of prolate oscillation is affected by the Reynolds number. In order to understand the impact of a liquid drop into a dry solid surface, Schroll et al. [5] use an axisymmetric volume-of-fluid (VOF) method to simulate the impact at reduced ambient pressure. Bernel et al. [6] experimentally and numerically investigated the drop formation by vortical flows in microgravity. Sun et al. [7] studied the deformation

\footnotetext{
* Corresponding author. Tel.: +86 10 82545792; fax: +86 1062541284

E-mail address: yangxf@imech.ac.cn (X. Yang)
}

and mass transfer for binary droplet collisions using the moving particle semi-implicit method.

The formation and deformation of liquid drops are associated with evolutionary morphology with changing free surfaces and moving interfaces, which present big challenges to numerical simulations. Over the past decades, interfacial flow simulation has been a formidable topic in computational fluid dynamics and computational physics. In order to deal with free surface and moving interface evolution, different numerical techniques have been proposed to track or capture free surfaces or moving interfaces. Considering the fact that both fluids and solids are composed of particles, the physics related liquid drop dynamics is closely related to the inter particle and intra molecular hydrodynamics interactions of the concerned multiple phase system. Therefore Lagrangian meshfree particle methods can be attractive in modeling liquid drops.

Smoothed particle hydrodynamics (SPH) is a typical meshfree particle method. It was first introduced by Lucy [8] and Gingold and Monaghan [9] for solving astrophysical problems, and later extended to many other problems in engineering and sciences [1013]. Nugent and Posch [14] firstly studied liquid drops and surface tension for van der Waals (vdW) fluids using SPH as the cohesive pressure part in the vdW equation of state can be used to model surface tension. Tartakovsky and Meakin [15] also developed an SPH method for modeling liquid drop dynamics with surface tension and contact angles for van der Waals fluids using an inter-particle interaction force. Xu et al. [16] developed a diffuse-interface 
SPH model which can also simulating vdW liquid-vapor systems. By introducing a surface tension model in SPH, Zhang [17] simulated binary collisions of liquid drops in two and three dimensions successfully. However, for higher Reynolds number and higher Weber number, the model has to be improved. Viscous droplet has also been studied using SPH method by many other researchers such as Jiang et al. [18] and Meleán et al. [19,20].

It is known that SPH suffers from tensile instability when modeling elastic solids and viscous fluids. The tensile instability is the situation in which when particles are under tensile stress state, the motion of the particle becomes unstable. It could result in particle clustering or particle blowing away in SPH computation. Swegle et al. [21,22] firstly studied the tensile instability and proposed a criterion which states that the instability depends on the sign of the multiplication of the stress and the second derivative of the kernel function. In SPH modeling viscous liquid drops, tensile instability also exist. Meleán et al. [19,20] investigated viscous liquid drops for vdW fluids, and discussed the tensile instability effects. In order to remove the tensile instability of SPH in modeling vdW drops, they applied the artificial stress model which was originally proposed by Monaghan [23] and is associated with an artificial viscous force and energy generation term. It is reported that with optimal coefficients, the artificial viscous stress model can remove tensile instability with sufficiently smoothed density profile.

This paper presents an improved SPH method for modeling viscous liquid drop without tensile instability using a hyperbolicshaped kernel function which possesses non-negative second derivatives. The paper is organized as follows. In the next section, SPH method for describing van der Waals fluid shall be briefly addressed. In Section 3, the SPH tensile instability shall be discussed and a hyperbolic-shaped kernel function shall be presented to remove tensile instability for SPH modeling viscous liquid drops. In Section 4, three classes of numerical examples shall be provided. The first class involves liquid drop formation both in 2D and 3D, the second class involves oscillation of liquid drops, and the third class involves head-on and off-center binary collisions of two vdW liquid drops. The paper concludes in Section 5 with some discussions.

\section{SPH method for van der Waals fluid}

In general, SPH is a numerical method for solving partial differential equations of continuum dynamics by replacing the continuum with a set of particles. In this section, we will briefly describe the governing equations of van der Waals (vdW) fluid and the corresponding SPH formulations.

In vdW flow, the effect of heat conduction is very important, so an internal energy equation should be considered. The general equations governing the motion of heat conducting and viscous fluid can be written as

$\frac{d \rho}{d t}=-\rho \nabla \cdot \boldsymbol{u}$

$\frac{d \boldsymbol{u}}{d t}=\frac{1}{\rho} \nabla \cdot \boldsymbol{S}+\mathbf{g}$

$\frac{d e}{d t}=\frac{1}{\rho} \boldsymbol{S}: \nabla \boldsymbol{u}-\frac{1}{\rho} \nabla \cdot \boldsymbol{q}$

where $t$ is time instant, $\rho$ is the density, $\boldsymbol{u}$ is the velocity, $\boldsymbol{S}$ is the stress tensor, $\boldsymbol{g}$ is the body force per unit mass, $e$ is the specific internal energy, and $\boldsymbol{q}$ is the heat flux vector.

The stress tensor $\boldsymbol{S}$ is written as

$\boldsymbol{S}=-p \boldsymbol{I}+\sigma$ where $p$ is the internal pressure, $\boldsymbol{I}$ is the unit tensor, and $\sigma$ is the viscous stress tensor given by

$\sigma=\eta(\nabla \boldsymbol{u}+\boldsymbol{u} \nabla)+\left(\zeta-\frac{2}{d} \eta\right)(\nabla \cdot \boldsymbol{u}) \boldsymbol{I}$

where $\eta$ and $\zeta$ is the coefficients of shear and bulk viscosity, respectively. The parameter $d$ is the spatial dimension, with $d=2$ and 3 for two- and three-space dimensions, respectively [20,24].

The heat flux vector is written as

$\mathbf{q}=-\kappa \nabla T$

where $\kappa$ is the coefficient of thermal conductivity and $T$ is the fluid temperature.

Eqs. (1)-(3) are closed by the mechanical and caloric equations of state for the pressure and the internal energy, respectively. For $\mathrm{vdW}$ fluid, the governing equations are closed by

$p=\frac{\rho \bar{k} T}{1-\rho \bar{b}}-\bar{a} \rho^{2}$

and

$e=\bar{k} T-\bar{a} \rho$

where $\bar{k}=k_{B} / m, \bar{a}=a / m^{2}$, and $\bar{b}=b / m$, here $k_{B}$ is the Boltzmann's constant, $m$ is the particle mass, $a$ is the cohesive action responsible for the short-range attractive forces between neighboring molecules, and $b$ is a constant parameter due to the finite size of the molecules. The cohesive part of the pressure in Eq. (7) gives rise to an attractive, central force between particles with an interaction range which is assumed to exceed that of all other smoothed forces appearing in Eq. (10). With this assumption, stable drops can be formed [20].

In SPH method, a continuous field is represented by a set of particles. The particles carry physical properties such as mass $m$, density $\rho$, velocity $\boldsymbol{u}$, and energy $e$. The governing equations are solved on particles and they can be written in SPH as $[14,19,20]$

$\frac{d \rho_{a}}{d t}=\sum_{b} m_{b}\left(\boldsymbol{u}_{a}-\boldsymbol{u}_{b}\right) \cdot \nabla_{a} W_{a b}$

$\frac{d \boldsymbol{u}_{a}}{d t}=\sum_{b} m_{b}\left(\frac{\boldsymbol{S}_{a}}{\rho_{a}^{2}}+\frac{\boldsymbol{S}_{b}}{\rho_{b}^{2}}\right) \cdot \nabla_{a} W_{a b}+\mathbf{g}_{a}$

$\begin{aligned} \frac{d e_{a}}{d t} & =\frac{1}{2} \sum_{b} m_{b}\left(\frac{\boldsymbol{S}_{a}}{\rho_{a}^{2}}+\frac{\boldsymbol{S}_{b}}{\rho_{b}^{2}}\right) \\ & :\left(\boldsymbol{u}_{a}-\boldsymbol{u}_{b}\right) \nabla_{a} W_{a b}-\sum_{b} m_{b}\left(\frac{\boldsymbol{q}_{a}}{\rho_{a}^{2}}+\frac{\boldsymbol{q}_{b}}{\rho_{b}^{2}}\right) \cdot \nabla_{a} W_{a b}\end{aligned}$

where subscripts $a$ and $b$ denote particle indexes, $W_{a b}=W\left(\boldsymbol{r}_{b}-\boldsymbol{r}_{b}\right.$, $h$ ) is a kernel function (also referred to as smoothing function, or simplified as kernel), $h$ is a smoothing length which determines the range of the interaction of particles, $\nabla_{a} W_{a b}$ denotes the gradient of the kernel function taken with respect to the coordinate of particle $a$. The summation is taken over all the neighboring particles determined by the smoothing length. Note that the spatial derivative in the governing equations has been transformed to the derivative of the kernel function which can be obtained analytically. The kernel function should satisfy some conditions which will be introduced in the following section.

In $\mathrm{SPH}$, an alternative method to approximate the density of a particle is

$\rho_{a}=\sum_{b} m_{b} W_{a b}$

There is a disadvantage that the density of the particles near free surface obtained by Eq. (12) always less than the reasonable value, 
because of the particle insufficiency effects near the free surface. Fortunately, this disadvantage is beneficial for the modeling of vdW liquid drops, because the fluid density near the free surface of a vdW liquid drop is less than the density far away the free surface. Therefore, Eq. (12) rather than Eq. (9) is used to calculate density in the follow numerical examples.

In modeling of vdW liquid drop [14], an frequently used SPH representation of the heat flux vector in Eq. (11) is written as

$\boldsymbol{q}_{a}=-\kappa \sum_{b} 2 m_{b} \frac{T_{b}-T_{a}}{\rho_{a}+\rho_{b}} \nabla_{a} W_{a b}$

Note that in order to calculate the divergence of the heat flux vector $\nabla \cdot \boldsymbol{q}$ the summation operation should be conducted twice (nested approximation). An alternative single-step approximation which is similar to the SPH heat conduction equation in reference [25] is

$$
\begin{aligned}
\left(\frac{1}{\rho} \nabla \cdot \boldsymbol{q}\right)_{a} & =\left(\frac{1}{\rho} \nabla \cdot(-\kappa \nabla T)\right)_{a} \\
& =-\kappa \sum_{b} \frac{2 m_{b}\left(\boldsymbol{r}_{b}-\boldsymbol{r}_{a}\right) \cdot \nabla_{a} W_{a b}}{\rho_{a} \rho_{b}\left(r_{a b}^{2}+\varepsilon\right)}\left(T_{b}-T_{a}\right)
\end{aligned}
$$

where $\varepsilon=0.01 h^{2}$ is used to prevent a singularity when $r_{a b}=0$. This single-step approximation for heat flux needs less computational efforts than the nested approximation and it will be demonstrated later that by using this single-step approximation, the obtained temperature field is smoother and more uniform. Therefore, Eq. (11) can be replaced by the follow formula is this paper as

$$
\begin{aligned}
\frac{d e_{a}}{d t}= & \frac{1}{2} \sum_{b} m_{b}\left(\frac{\boldsymbol{S}_{a}}{\rho_{a}^{2}}+\frac{\boldsymbol{S}_{b}}{\rho_{b}^{2}}\right): \boldsymbol{u}_{a b} \nabla_{a} W_{a b} \\
& +\kappa \sum_{b} \frac{2 m_{b}\left(\boldsymbol{r}_{b}-\boldsymbol{r}_{a}\right) \cdot \nabla_{a} W_{a b}}{\rho_{a} \rho_{b}\left(r_{a b}^{2}+\eta\right)}\left(T_{b}-T_{a}\right)
\end{aligned}
$$

According to [14], in order to obtain stable drops, the interaction range of the cohesive pressure of the vdW equation of state is assumed to exceed the interaction range of all the other forces in the SPH equations of motion. In this assumption, the cohesive pressure $-\bar{a} \rho^{2}$ should be considered separately. The corresponding SPH formulations are written as

$$
\left(\frac{d \boldsymbol{u}_{a}}{d t}\right)^{c p}=2 \bar{a} \sum_{b} m_{b} \nabla_{a} W_{a b}^{H}
$$

$$
\left(\frac{d e_{a}}{d t}\right)^{c p}=\bar{a} \sum_{b} m_{b}\left(\boldsymbol{u}_{b}-\boldsymbol{u}_{a}\right) \cdot \nabla_{a} W_{a b}^{H}
$$

where the subscript $c p$ denotes the term contributed by the cohesive pressure, and $H$ denotes the corresponding smoothing range of the cohesive pressure. In this paper we take $H=2 h$ as in [14].

\section{SPH tensile instability and kernel functions}

It is well known that SPH simulations may suffer from tensile instability which usually causes particle clustering or blowing away. The tensile instability is frequently observed in SPH modeling of solid [22,26], and it is also observable in fluid modeling even when the pressure is positive [27]. Several remedies have been proposed to improve or avoid such tensile instability. For example, in order to remove the instability in positive pressure state, Schussler and Schmitt [27] suggested a kernel that gives short range repulsion. However, their kernel function is not sufficiently smooth and its second derivative is not continuous; numerical simulations show that smoother kernels give better kernel approximations when the particles are disordered [26]. There have been many other approaches to remove the tensile instability [23,28-35].
These approaches can be effective in some cases, and none of them are successful for all cases $[26,36]$.

The tensile instability was first studied in detail by Swegle et al. $[21,22]$ through a one-dimensional von Neumann stability analysis. They proposed a criterion for being stable or instable in terms of the stress state and the second derivative of the smoothing kernel function, i.e., a sufficient condition for the unstable growth is

$W^{\prime \prime} S>0$

where $W^{\prime \prime}$ is the second derivative of the kernel function, and $S$ is the stress state. Therefore, the frequently named "tensile instability" should be rigorously referred to as "stress instability".

In standard SPH, bell-shaped kernels are frequently used. The second derivative of a bell-shaped kernel, such as the cubic spline (see Fig. 1), changes sign from negative to positive so that the product of the stress times the second derivative of the kernel function will changes sign whether the stress is positive or negative. For example, for the commonly used cubic spline kernel function, it can be expressed as follows

$W_{B}(s, h)=\alpha_{d} \begin{cases}(2-s)^{3}-4(1-s)^{3}, & 0 \leq s<1 \\ (2-s)^{3}, & 1 \leq s<2 \\ 0, & s \geq 2\end{cases}$

where $s=r / h, \alpha_{d}$ is the normalization factor, with the value of $1 / 6 h$, $5 /\left(14 \pi h^{2}\right)$ and $1 /\left(4 \pi h^{3}\right)$ in one, two and three dimensional space, respectively. As can be seen in Fig. 1, there exist instable region whether the stress is positive or negative. As the smoothing length, $h$, in the SPH model is usually equal or slightly bigger than particle spacing, SPH particles generally locate in region $(s \geq 1)$, where the second derivatives of the kernel function is non-negative, and SPH computation is unstable under tensile stress state (i.e., tensile instability).

As the second derivative of a bell-shaped kernel changes sign in the support domain $(0 \leqslant s \leqslant 2)$, it is hardly inevitable to remove stress instability by simply using a bell-shaped kernel. In order to remove tensile instability when the pressure is positive, a hyperbolic-shaped kernel is used in this paper as follows [37]

$W_{H}(s, h)=\alpha_{d} \begin{cases}s^{3}-6 s+6, & 0 \leq s<1 \\ (2-s)^{3}, & 1 \leq s<2 \\ 0, & 2 \leq s\end{cases}$

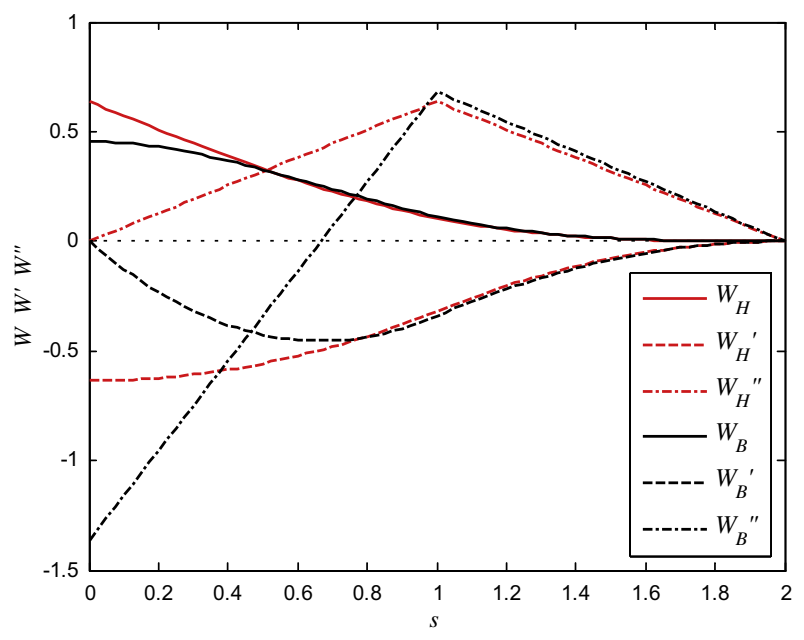

Fig. 1. Kernel functions and their first two derivatives. $W_{H}$ : the new hyperbolicshaped kernel; $W_{B}$ : the cubic spline kernel. 
where $\alpha_{d}$ is $1 /(7 h), 1 /\left(3 \pi h^{2}\right)$ and $15 /\left(62 \pi h^{3}\right)$ in one, two and three dimensional space, respectively. Similar to bell-shaped kernel functions, this new hyperbolic-shaped kernel satisfies some general requirements, such as normalization condition, Delta function behavior and compact supportness [38].

The new hyperbolic-shaped kernel and the cubic spline kernel are comparatively shown in Fig. 1. A significant difference between them is that the second derivative of the hyperbolic-shaped kernel function is non-negative in the entire support domain. This ensures that the repulsive force between SPH particles will not decrease when particles approach each other in compression. It is noted that as the second derivative of this kernel is non-negative, if SPH particles are under negative stress, the corresponding SPH simulation is stable according to Eq. (18). As the stress is negative (positive pressure) in viscous fluids, SPH modeling of viscous fluid dynamic problems such as viscous drop dynamics by using this new hyperbolic-shaped kernel function is expected to be stable.

\section{Numerical results}

Using the SPH method described in previous sections, a series of numerical modeling of van der Waals liquid drops are performed in this section. In all the simulations, the SPH particles are of the same mass $m$. The parameters in the equations of state are adopted the same as employed by Nugent and Posch [14], namely, $m=1$, $\bar{a}=2, \bar{b}=0.5$, and $\bar{k}=1$, here reduced units are used. The coefficient of thermal conductivity is $\kappa=5$.

\subsection{Formation of $v d W$ drops}

In the absence of any external force, a liquid drop will form a sphere due to the existence of surface tension. As an example, the formation of a 3D vdW liquid drop is firstly simulated. 27,000 particles are initially arranged on a cube (see Fig. 2) and the initial particle spacing is 0.85 . The shear and bulk viscosities are 1.0 and 0.1 , respectively. The time step is 0.005 . The initial temperature is 0.2. All quantities are given in reduced units.

Fig. 3 shows a sequence of snapshots for the formation of a 3D vdW drop. In the simulation, the cubic spline kernel function Eq. (19) and the hyperbolic-shaped kernel function Eq. (20) are used,

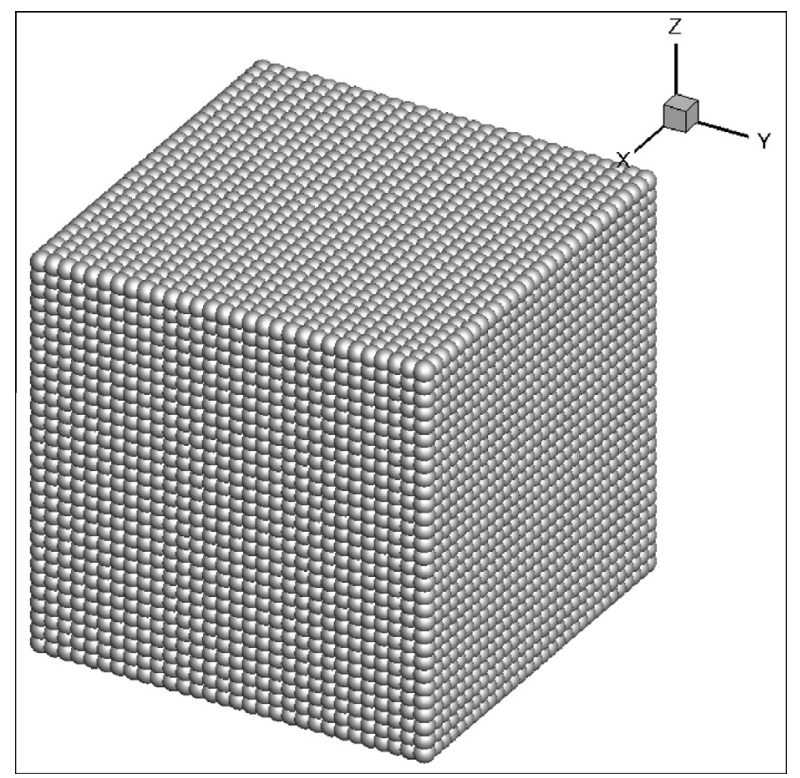

Fig. 2. The initial particle configuration for a 3D vdW liquid drop formation. respectively. For both kernel functions, the initially cube-shaped bulk liquid is rounded off at the cubic corners, gradually turns to be spherical, and finally produces a stable liquid sphere. However, the SPH snapshots obtained using the cubic spline functions are quite rough with particle clustering both in the surface area and in the interior region. This is an obvious demonstration of tensile instability. In contrast, using the hyperbolic-shaped kernel function, SPH particles are distributed more uniformly without particle clustering. It is clear that using the hyperbolic-shaped kernel function removes the tensile instability in viscous liquid drop.

If SPH particles are arranged in a square in 2D, a stable circular drop will also be formed due to the effect of surface tension. As 2D simulations need less SPH particle with smaller computational cost and it is also more convenient for observing the internal structure of SPH particle distribution, following examples including liquid drop formation, oscillation and collisions are all conducted in 2D spaces. For the case of $2 \mathrm{D}$ vdW viscous liquid drop formation, 900 particles are initially arranged on a square (see Fig. 4) and of the same temperature $T=0.2$. The initial particle spacing is 0.75 and the time step is set to be 0.005 . The coefficients of shear and bulk viscosities are $\eta=1$ and $\zeta=0.1$, respectively.

Fig. 5 shows the SPH particle evolution during a 2D viscous liquid drop formation using the cubic spline kernel (left column) and the hyperbolic-shaped kernel function (right column) at $t=5,10,50$ and 500. Again for both kernel functions, the initially square-shaped bulk liquid is rounded off at the square corners, gradually turns to be circular, and finally produces a stable liquid circle. It is clear that for the cubic spline kernel, the corresponding SPH snapshots demonstrate obvious tensile instability. SPH particles are clustered both in radial and circumferential directions, and therefore during the evolution process, particles are not evenly distributed. This can also lead to local particle number density fluctuations with non-smoothed density and pressure fields. In contrast, for the hyperbolicshaped kernel, the corresponding SPH snapshots show no tensile instability. SPH particles are distributed more uniformly without particle clustering. The final particle distribution of the stable liquid drop is more reasonable without local particle number density fluctuations.

As discussed in Section 2 that heat flux is important in modeling $\mathrm{vdW}$ viscous liquid drop and different approaches in modeling heat flux can lead to different behavior of the liquid drop. Fig. 6 shows the temperature field for a stable liquid drop obtained using the nested and single step approximations for heat flux expressed in Eqs. (11) and (14) at $t=500$. It is clear that by using the single step SPH approximations for heat flux, the obtained temperature field is smoother and more uniform.

Figs. 7 and 8 show the density and pressure profiles of the final stable circular liquid drop. It can be seen that in Fig. 7 the density is nearly of the same except in the area near the free surface of the drop. In Fig. 8, the pressure near the free surface is very low and negative. The surface layer of negative pressure results in surface tension.

It is possible to calculate surface tension from the SPH simulation results for viscous liquid drops considering the Lapalce equation. For a stable liquid drop, the Laplace equation is satisfied [14]

$p_{l}=p_{g}+\frac{\gamma}{R}$

where $\gamma$ denotes the surface tension, $p_{l}$ denotes the pressure in the drop center, $p_{g}$ denotes the vapor pressure far away from the drop, and $R$ is the radius of the drop.

A number of viscous liquid drops with different radii are simulated. The pressure at the center of the drop versus $1 / R$ is shown in Fig. 9. The pressure can be fitted as the following line 


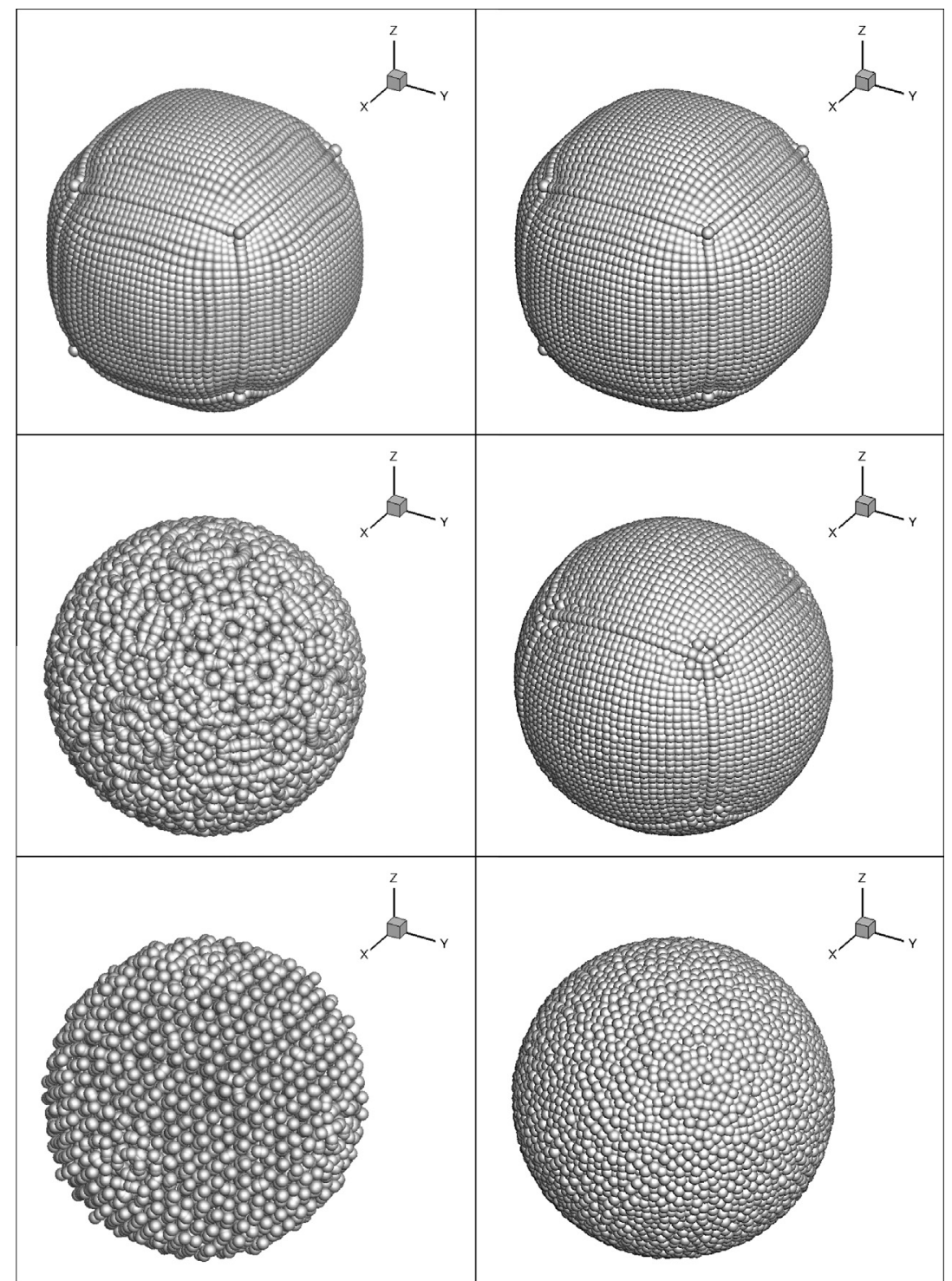

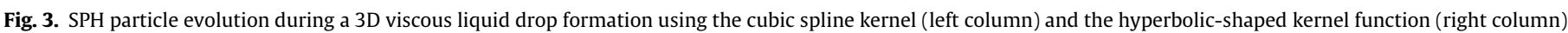
(from top to bottom $t=10,50,200$ ).

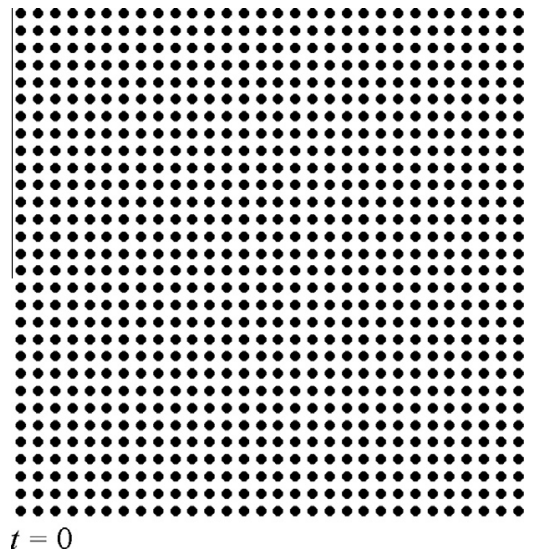

Fig. 4. The initial particle configuration for a $2 \mathrm{D} v \mathrm{vW}$ liquid drop formation.

$p_{l}=0.067+\frac{3.2}{R}$
From Eqs. (21) and (22), we find $\gamma_{L}=3.2$, here the subscript $L$ denotes the surface tension is obtained from the Laplace Eq. (21).

\subsection{Oscillation of $v d W$ drops}

In this section, we shall investigate the small-amplitude oscillation of a $2 \mathrm{D}$ vdW liquid drop using the hyperbolic-shaped kernel function and the single-step approximation for heat flux. After a 2D vdW liquid drop reaches equilibrium, the circular drop was transformed into an oval drop by the following conversion formula [15]

$$
\left(\begin{array}{l}
x^{*} \\
y^{*}
\end{array}\right)=\sqrt{\frac{2}{\sin \theta}}\left[\begin{array}{l}
x \sin (\theta / 2) \\
y \cos (\theta / 2)
\end{array}\right]
$$

where $\theta=\varepsilon \pi$, the eccentricity $\varepsilon=0.55$. When the oval drop is released, it will do oscillatory motion due to the surface tension.

Fig. 10 shows a sequence of snapshots for the oscillation of the 2D vdW drop. Again, SPH results show no tensile instability and $\mathrm{SPH}$ particles are distributed more uniformly without particle 


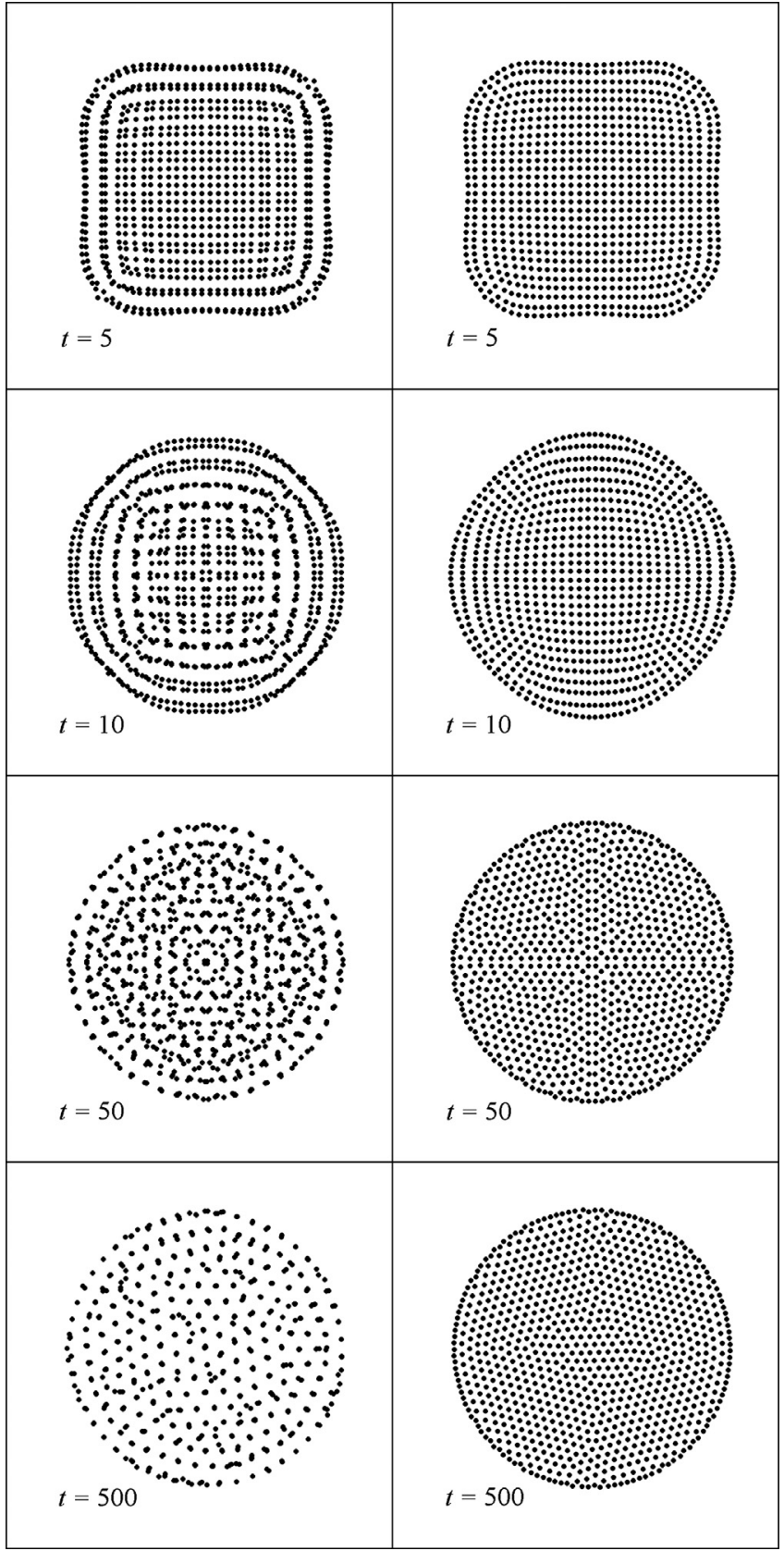

Fig. 5. SPH particle evolution during a $2 \mathrm{D}$ viscous liquid drop formation using the cubic spline kernel (left column) and the hyperbolic-shaped kernel function (right column) (from top to bottom $t=5,10,50,500$ ).

clustering. Fig. 11 shows the radius along the $x$ and $y$ directions versus time. It is clear that the oscillation and decay process of the liquid drop are closely related to shear and bulk viscosities used in the SPH simulation. For shear viscosity 1.0 and the bulk viscosity 0.1 , the amplitude of the oscillation decays quickly (Fig. 11). If turning off the shear and bulk viscosities, the amplitude of the oscillation decays much slower as shown in Fig. 12. During the entire oscillation process, SPH particles are distributed evenly without local particle clustering, and tensile instability does not exist.

It is also feasible to calculate surface tension from the small oscillation of liquid drops. In the classical work by Rayleigh [2], for small vibration amplitudes, the normal-model frequencies $\omega$ are related to the surface tension. For 2D oscillation of drops, the period of oscillations $\tau(=2 \pi / \omega)$ can be obtained from $[2,14]$

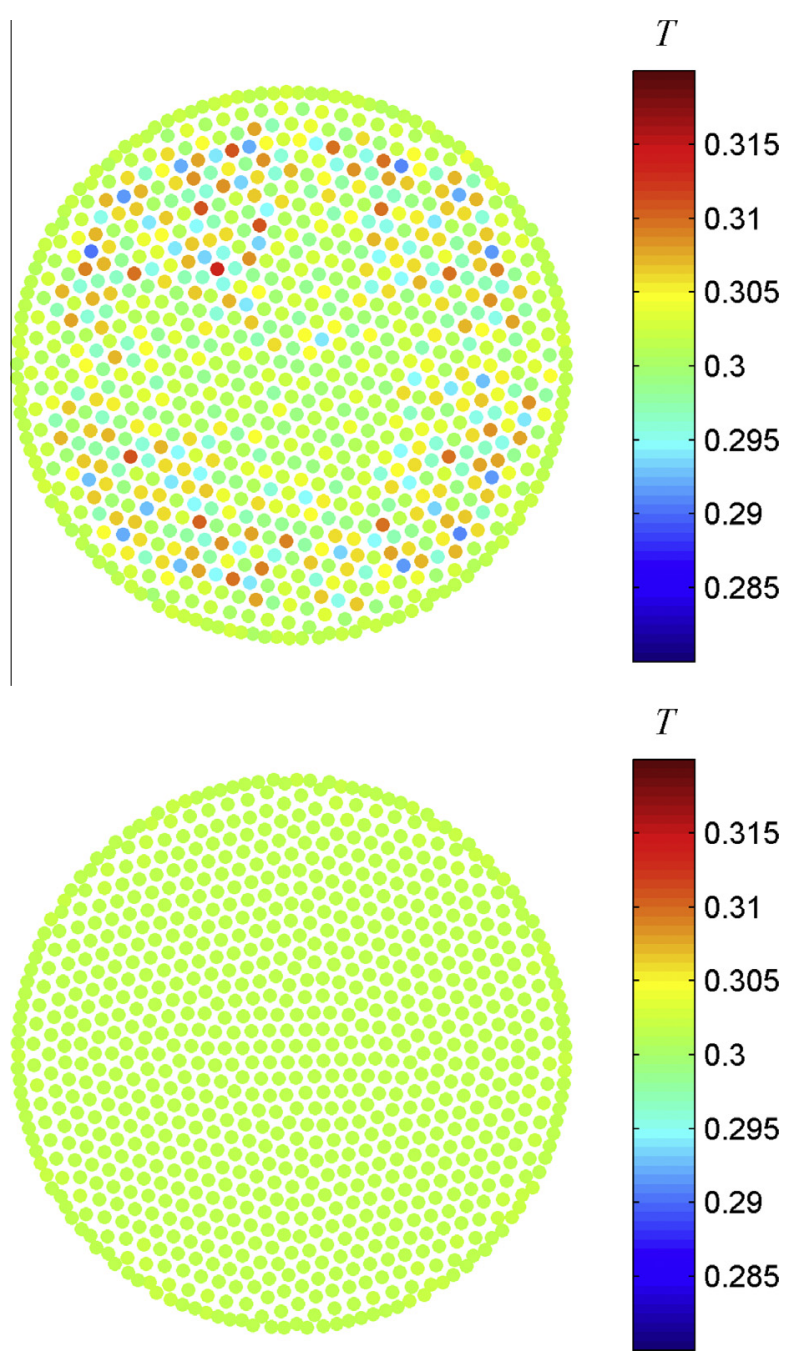

Fig. 6. Temperature fields for the $2 \mathrm{D}$ stable liquid drop obtained using the nested approximation (top) and the single-step approximation (bottom) for heat flux $(t=500)$.

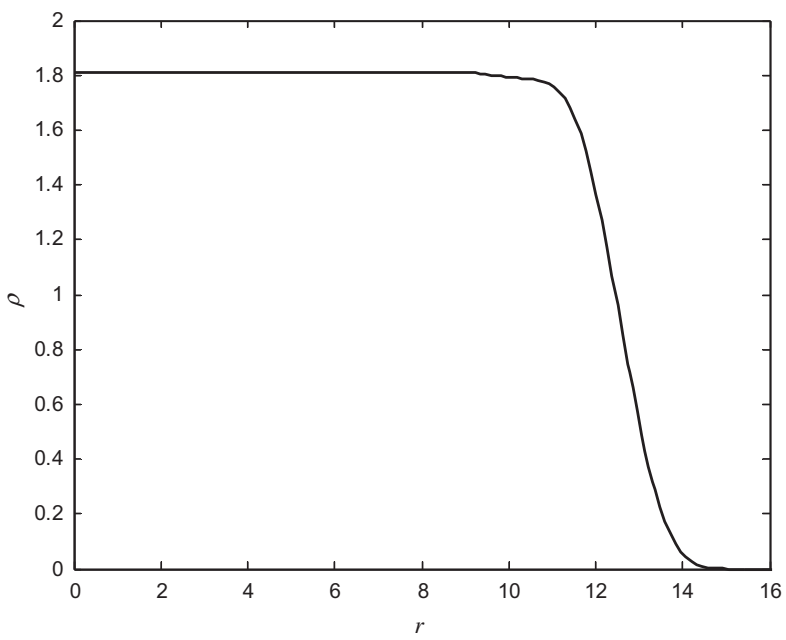

Fig. 7. Smoothed density profile of a $2 \mathrm{D}$ vdW drop as a function of radius $(t=500)$.

$$
\tau=2 \pi \sqrt{\frac{\rho R^{3}}{6 \gamma}}
$$




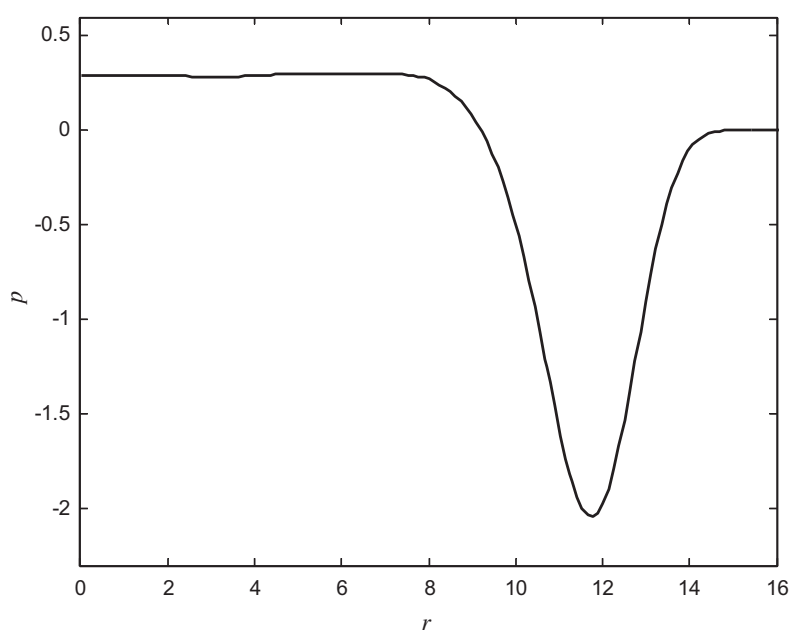

Fig. 8. Smoothed pressure profile of a vdW drop as a function of radius $(t=500)$.

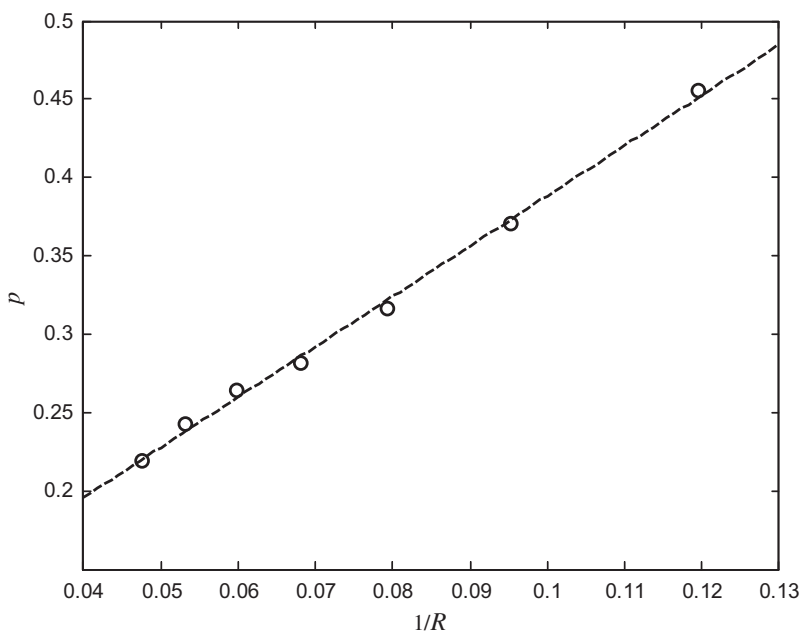

Fig. 9. The pressure at the center of vdW drops for various drop radii. "o" denotes the pressure at the center of the drop versus $1 / R$; “-" denotes the fitted line.

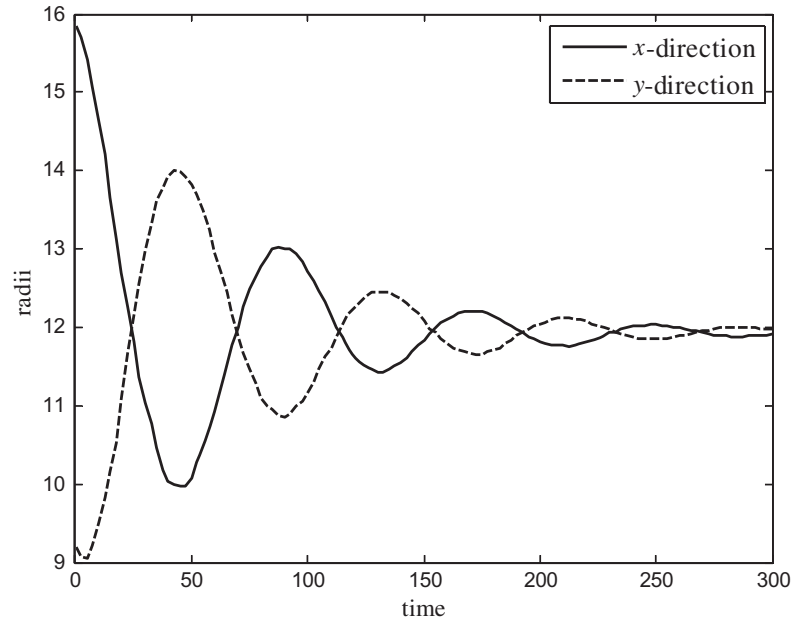

Fig. 11. Oscillations of a vdW drop with viscosity with the shear viscosity 1.0 and the bulk viscosity 0.1 .

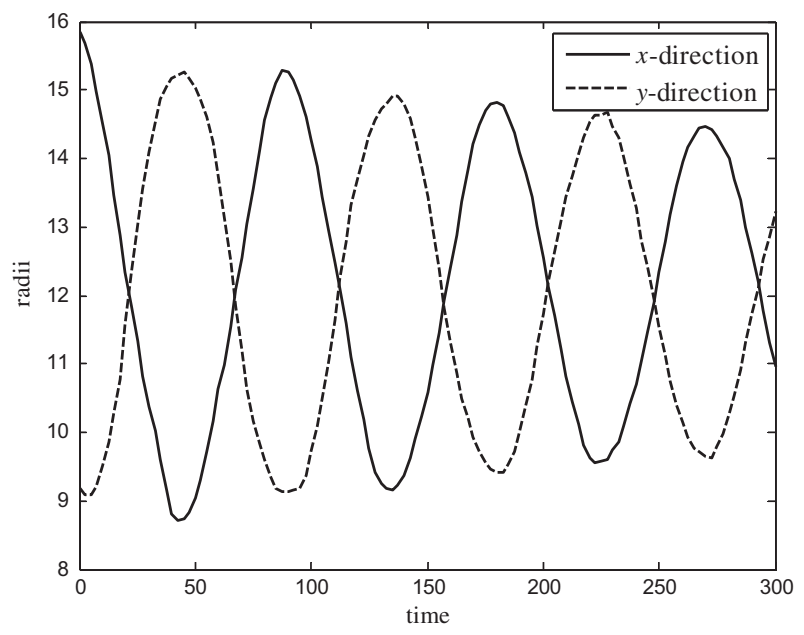

Fig. 12. Oscillations of a vdW liquid drop with both the shear and the bulk viscosities equal to 0 .

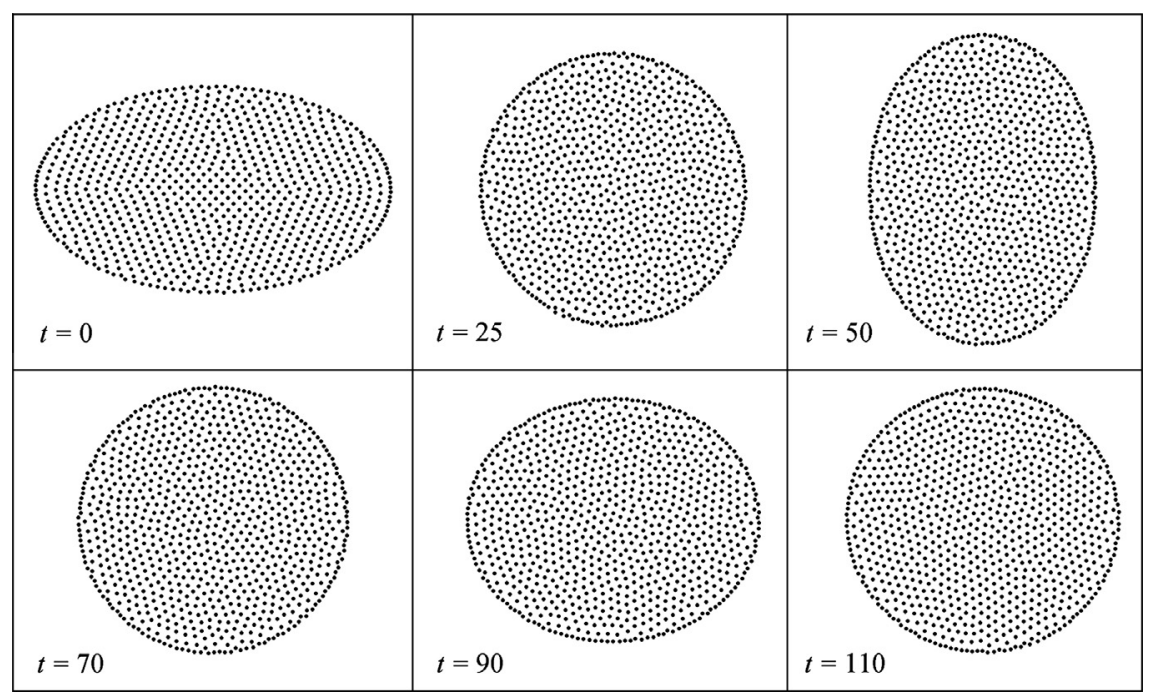

Fig. 10. Oscillation process of a $2 \mathrm{D}$ vdW drop. 


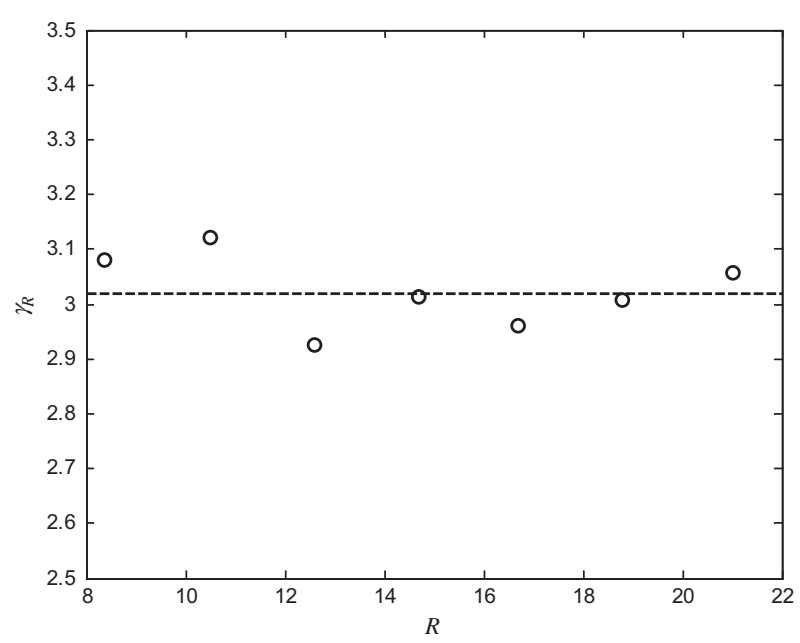

Fig. 13. The surface tension for various drop radii. "o" denotes the surface tension obtained from Eq. (25); “-” denotes the mean value of the surface tension for various drop radii.

where $R$ is the equilibrium radius of the drop and $\rho$ is the density of the fluid in the drop. From Eq. (24) we can find that

$\gamma_{R}=\frac{2 \pi^{2} \rho R^{3}}{3 \tau^{2}}$

where the subscript $R$ denotes the surface tension is obtained from the work done by Rayleigh [2].

Fig. 13 shows the surface tension $\gamma_{R}$ obtained from Eq. (25) for various drop radii. The surface tensions $\gamma_{R}$ and $\gamma_{L}$ obtained from Eq. (21) agree with each other, with a relative discrepancy $\left(\left|\gamma_{R}-\gamma_{L}\right| / \gamma_{L} \times 100 \%\right)$ smaller than $10 \%$. The agreement of surface tensions obtained by these two different examples demonstrates that vdW liquid drops and surface tension can be effectively modeled by the modified SPH method.

\subsection{Binary collision of $v d W$ drops}

In this section, head-on and off-center collisions of two viscous liquid drops are simulated. The initial drops are the copies of the 2D drop obtained in Section 3, which are at equilibrium state. The relative velocity of two drops is 0.2 .

For head-on collision, two drops have the same speed of 0.1 , move in opposite directions along the same straight line, collide and merge with each other. Fig. 14 shows a sequence of snapshots of head-on collision of 2D vdW drops. At the initial stage when two drops meet each other, a liquid bridge forms between them, then a dumbell-shaped drop was produced. The width of the liquid bridge become bigger and bigger, then the two drop fully merged into a bigger liquid drop. Due to the surface tension effects, the liquid drop oscillated a number of periods, similar to the drop oscillation in Section 4.1.

For the 2D Off-center collision, the relative velocity of two drops is 0.2 . The distance between the centers of the two drops in the direction perpendicular to the velocity direction equals to the radii of the drop. Fig. 15 shows the particle evolution process during the off-center collision. Again, as the two liquid drops approach each other, a liquid bridge and a dumbbell-shaped liquid drop are produced. The merged bigger liquid drop oscillated along the initial centerline of the two initial drops for a number of periods and then reaches steady state. It is noted that for both head-on and off-center collusions, SPH particles are distributed evenly without local particle clustering. This also shows that tensile inutility has been effectively removed from the SPH simulation using the hyperbolic-shaped kernel function.

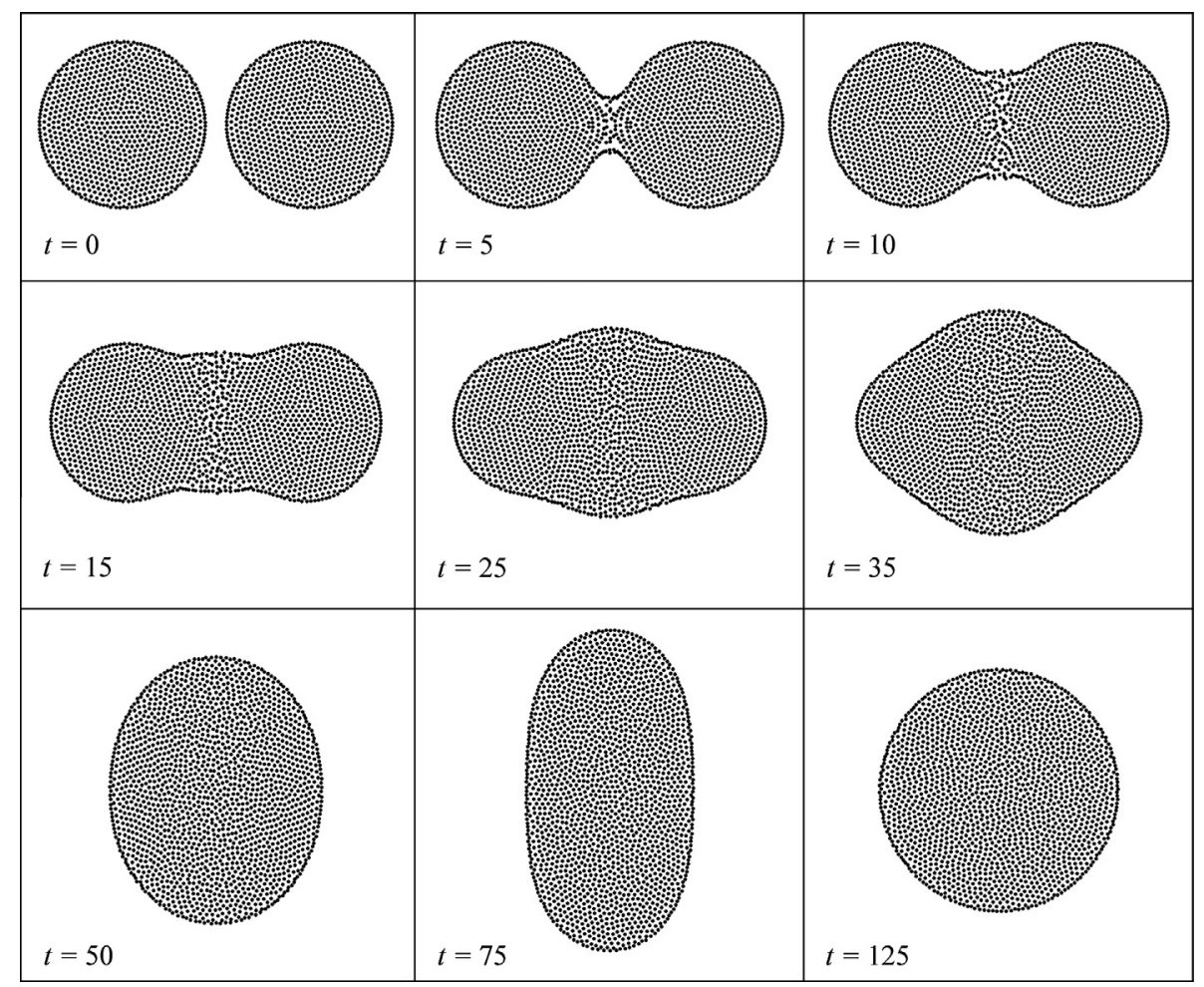

Fig. 14. 2D head-on binary collision with relative velocity of 0.2 . 


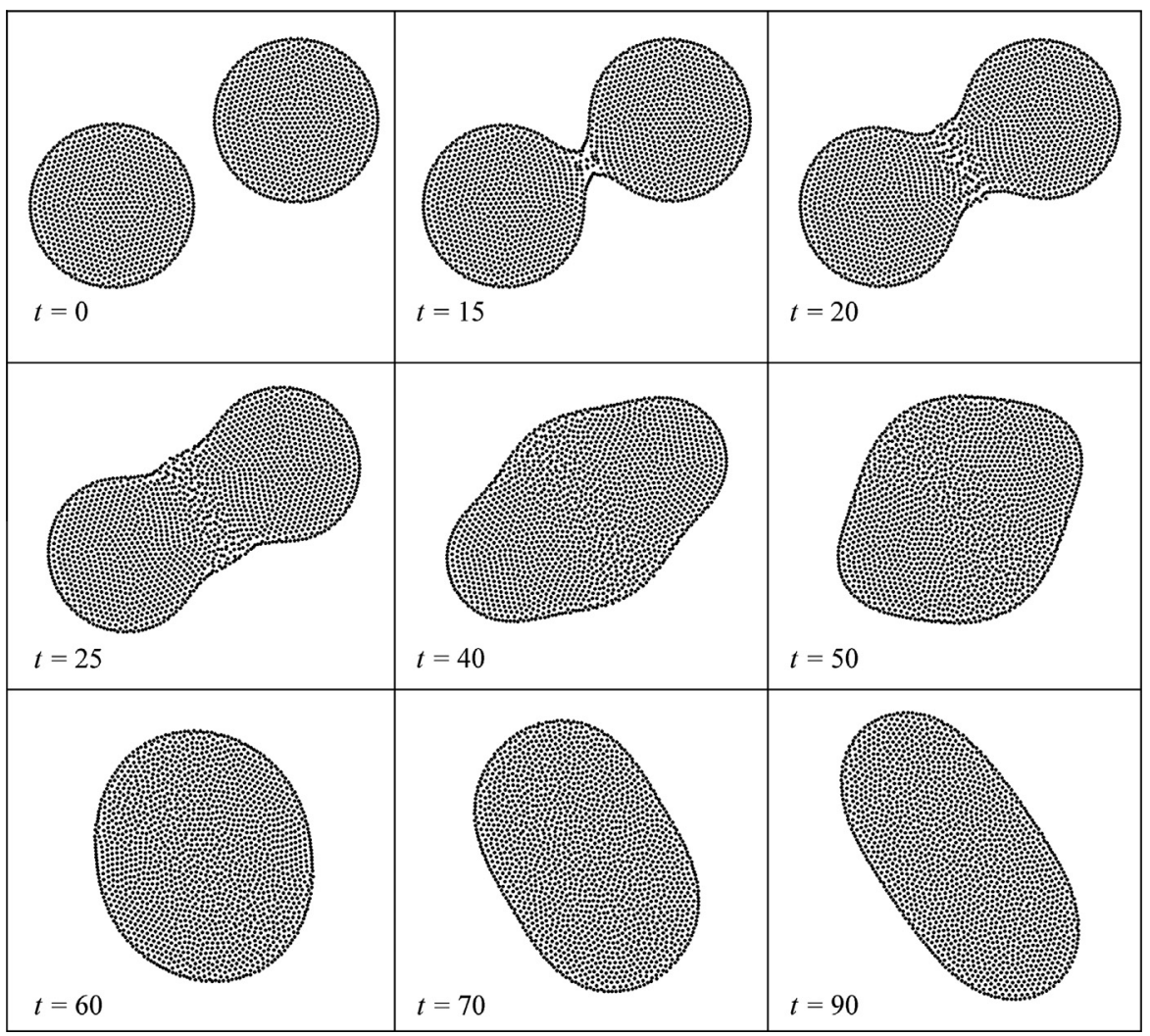

Fig. 15. 2D off-center binary collision with relative velocity of 0.2 .

\section{Conclusion}

Conventional SPH methods for modeling viscous liquid drop dynamics are associated with tensile instability, which shows unphysical particle clustering with varying local number density, and rough pressure and temperature fields. This paper presents the numerical simulations of viscous liquid drop dynamics with a modified SPH method with a hyperbolic-shaped kernel function. The newly proposed hyperbolic-shaped kernel function satisfies common requirements for general kernel functions, and has nonnegative second order derivatives, such as normalization condition, Delta function behavior and compact supportness. However, different from frequently used bell-shaped kernel functions, the second derivative of the hyperbolic-shaped kernel function is non-negative in the entire support domain. According to the sufficient condition for the unstable growth, a kernel function with non-negative second derivates is stable for SPH simulations under negative stresses. Therefore SPH modeling of viscous fluid dynamic problems using this hyperbolic-shaped kernel function are associated with evenly distributed particles without tensile instability, as shown in conventional SPH simulations. In treating the heat flux in modeling viscous liquid drop, a single-step approximation scheme is used. Compared to the commonly used nested approximation scheme, this single-step approximation needs less computational efforts with smoother and more uniform temperature field.

Three numerical examples including formation, oscillation and collisions of liquid drops are simulated using this modified SPH method with the new hyperbolic-shaped kernel function and single-step approximation scheme for heat flux. Surface tension is calculated for both the formation and oscillation of liquid drops with a small discrepancy. More importantly, the obtained SPH results show even particle distributions without tensile instability. It is demonstrated that this modified SPH method can effectively model viscous drop dynamics.

\section{Acknowledgement}

This work has been supported by the National Natural Science Foundation of China (11302237 and 11172306).

\section{References}

[1] Willis K, Orme M. Binary droplet collisions in a vacuum environment: an experimental investigation of the role of viscosity. Exp Fluids 2003;34:28-41.

[2] Rayleigh L. On the capillary phenomena of jets. Proc R Soc London 1879;29:71-97.

[3] Apfel RE, Tian Y, Jankovsky J, Shi T, Chen X, Holt RG, et al. Free oscillations and surfactant studies of superdeformed drops in microgravity. Phys Rev Lett 1997; 78:1912-5

[4] Willis KD, Orme ME. Experiments on the dynamics of droplet collisions in a vacuum. Exp Fluids 2000;29:347-58.

[5] Schroll RD, Josserand C, Zaleski S, Zhang WW. Impact of a viscous liquid drop. Phys Rev Lett 2010;104:034504.

[6] Bernal LP, Maksimovic P, Tounsi F, Tryggvason G. An experimental and numerical investigation of drop formation by vortical flows in microgravity. In: 32nd Aerospace sciences meeting and exhibit Reno. NV: American Institute of Aeronautics and Astronautics; 1994.

[7] Sun Z, Xi G, Chen X. Mechanism study of deformation and mass transfer for binary droplet collisions with particle method. Phys Fluids 2009;21:032106.

[8] Lucy LB. A numerical approach to the testing of the fission hypothesis. Astron J 1977;82:1013-24.

[9] Gingold RA, Monaghan JJ. Smoothed particle hydrodynamics: theory and application to non-spherical stars. Mon Not R Astron Soc 1977;181:375-89.

[10] Liu MB, Liu GR. Smoothed particle hydrodynamics (SPH): an overview and recent developments. Arch Comput Methods Eng 2010;17:25-76.

[11] Ferrari A, Dumbser M, Toro EF, Armanini A. A new 3D parallel SPH scheme for free surface flows. Comput Fluids 2009;38:1203-17.

[12] Breinlinger T, Polfer P, Hashibon A, Kraft T. Surface tension and wetting effects with smoothed particle hydrodynamics. J Comput Phys 2013;243:14-27.

[13] Monaghan J. Smoothed particle hydrodynamics and its diverse applications. Annu Rev Fluid Mech 2012;44:323-46. 
[14] Nugent S, Posch HA. Liquid drops and surface tension with smoothed particle applied mechanics. Phys Rev E 2000;62:4968-75.

[15] Tartakovsky A, Meakin P. Modeling of surface tension and contact angles with smoothed particle hydrodynamics. Phys Rev E 2005;72:026301.

[16] Xu Z, Meakin P, Tartakovsky AM. Diffuse-interface model for smoothed particle hydrodynamics. Phys Rev E 2009;79:036702.

[17] Zhang M. Simulation of surface tension in 2D and 3D with smoothed particle hydrodynamics method. J Comput Phys 2010;229:7238-59.

[18] Jiang T, Ouyang J, Zhao X, Ren J. The deformation process of viscous liquid drop studied by using kernel gradient corrected SPH method. Acta Phys Sin 2011;60:054701.

[19] Meleán Y, Sigalotti LDG. Coalescence of colliding van der Waals liquid drops. Int J Heat Mass Transf 2005;48:4041-61.

[20] Meleán Y, Sigalotti LDG, Hasmy A. On the SPH tensile instability in forming viscous liquid drops. Comput Phys Commun 2004;157:191-200.

[21] Swegle JW, Hicks DL, Attaway SW. Smoothed particle hydrodynamics stability analysis. J Comput Phys 1995;116:123-34.

[22] Swegle JW, Attaway SW, Heinstein MW, Mello FJ, Hicks DL. An analysis of smoothed particle hydrodynamics. Albuquerque (NM, United States): Sandia National Labs; 1994

[23] Monaghan JJ. SPH without a tensile instability. J Comput Phys 2000;159:290-311.

[24] Sigalotti LDG, Klapp J, Sira E, Meleán Y, Hasmy A. SPH simulations of timedependent Poiseuille flow at low Reynolds numbers. J Comput Phys 2003;191:622-38.

[25] Monaghan JJ. Smoothed particle hydrodynamics. Rep Prog Phys 2005;68:1703-59.

[26] Gray JP, Monaghan JJ, Swift RP. SPH elastic dynamics. Comput Methods Appl Mech Eng 2001;190:6641-62.
[27] Schuessler I, Schmitt D. Comments on smoothed particle hydrodynamics. Astron Astrophys 1981;97:373-9.

[28] Johnson GR, Beissel SR. Normalized smoothing functions for SPH impact computations. Int J Num Methods Eng 1996;39:2725-41.

[29] Morris JP. Analysis of smoothed particle hydrodynamics with applications. Monash University; 1996.

[30] Randles PW, Libersky LD. Smoothed particle hydrodynamics: some recen improvements and applications. Comput Methods Appl Mech Eng 1996;139:375-408.

[31] Chen JK, Beraun JE, Jih CJ. An improvement for tensile instability in smoothed particle hydrodynamics. Comput Mech 1999;23:279-87.

[32] Dyka CT, Ingel RP. An approach for tension instability in smoothed particle hydrodynamics (SPH). Comput Struct 1995;57:573-80.

[33] Dyka CT, Randles PW, Ingel RP. Stress points for tension instability in SPH. Int Num Methods Eng 1997;40:2325-41.

[34] Wen Y, Hicks DL, Swegle JW. Stabilizing SPH with conservative smoothing. Albuquerque (NM, United States): Sandia National Labs; 1994.

[35] Hicks DL, Liebrock LM. SPH hydrocodes can be stabilized with shape-shifting. Comput Math Appl 1999;38:1-16.

[36] Mandell DA, Wingate CA, Schwalbe LA. Computational brittle fracture using smooth particle hydrodynamics. NM, United States: Los Alamos National Laboratory; 1996.

[37] Yang XF, Liu MB. An improvement for stress instability in smoothed particle hydrodynamics (in Chinese). Acta Phys Sin 2012;61:224701.

[38] Liu MB, Liu GR, Lam KY. Constructing smoothing functions in smoothed particle hydrodynamics with applications. J Comput Appl Math 2003:155:263-84. 